LPT-ORSAY-10/96

CERN-PH-TH/2010-296

\title{
Revisiting the constraints on the Supersymmetric Higgs sector at the Tevatron
}

\author{
Julien Baglio ${ }^{1}$ and Abdelhak Djouadi ${ }^{1,2}$ \\ ${ }^{1}$ Laboratoire de Physique Théorique, Université Paris XI et CNRS, F-91405 Orsay, France. \\ 2 Theory Unit, CERN, 1211 Genève 23, Switzerland.
}

\begin{abstract}
We analyze the production of the neutral Higgs particles of the Minimal Supersymmetric extension of the Standard Model at the Fermilab Tevatron collider. We consider the two main production and detection channels: gluon-gluon and bottom quark fusion leading to Higgs bosons which subsequently decay into tau leptons, $g g, b \bar{b} \rightarrow \operatorname{Higgs} \rightarrow \tau^{+} \tau^{-}$. We update the production cross sections and the decay branching ratios and obtain production rates that are significantly smaller at high masses than the ones used by the CDF and D0 experiments in their search. We then evaluate the various theoretical uncertainties that affect these rates, uncertainties that have not been been considered in the CDF/D0 analyses and which turn out to be rather large. Including these two effects will significantly loosen the constraints obtained on the supersymmetric Higgs sector at the Tevatron.
\end{abstract}


The search for the Higgs bosons, the remnants of the spontaneous breaking of the electroweak symmetry that is at the origin of the elementary particle masses, is the main goal of present high-energy colliders. While a single Higgs boson is predicted in the Standard Model (SM), the minimal realization of the symmetry breaking with only one Higgs doublet field [1, the Higgs sector is extended in supersymmetric theories [2], that are widely considered to be the most attractive extensions of the SM as they stabilize the hierarchy between the electroweak and Planck scales induced by the large radiative corrections to the Higgs boson mass. In the minimal extension, the Minimal Supersymmetric Standard Model (MSSM) [2], two Higgs doublet fields are required, leading to the existence of five Higgs particles: two $\mathrm{CP}-$ even $h$ and $H$, a CP-odd $A$ and two charged $H^{ \pm}$particles [3,4].

In the MSSM, two parameters are needed to describe the Higgs sector at tree-level: the mass $M_{A}$ of the pseudoscalar boson and the ratio of vacuum expectation values of the two Higgs fields, $\tan \beta$, that is expected to lie in the range $1 \lesssim \tan \beta \lesssim 50$. At high $\tan \beta$ values, $\tan \beta \gtrsim 10$, one of the neutral CP-even states has almost exactly the properties of the SM Higgs particle: its couplings to fermions and gauge bosons are the same, but its mass is restricted to values $M_{h}^{\max } \approx 110-135 \mathrm{GeV}$ depending on some SUSY parameters that enter the radiative corrections [4]. The other $\mathrm{CP}$-even and the $\mathrm{CP}$-odd states, that we will denote collectively by $\Phi=A, H(h)$, are then almost degenerate in mass and have the same properties: no couplings to gauge bosons, while the couplings to isospin down-type (up-type) quarks and charged leptons are (inversely) proportional to $\tan \beta$.

Thus, for $\tan \beta \gtrsim 10$, the $\Phi$ boson couplings to bottom quarks and $\tau$-leptons are strongly enhanced while those to top quarks are suppressed. As a result, the phenomenology of these states becomes rather simple. To a very good approximation, the $\Phi$ bosons decay almost exclusively into $b \bar{b}$ and $\tau^{+} \tau^{-}$pairs with branching ratios of, respectively, $\approx 90 \%$ and $\approx 10 \%$, while the other decay channels are suppressed to a negligible level [5]. The main production mechanisms for these particles are those processes which involve the couplings to bottom quarks. At hadron colliders, these are the gluon-gluon fusion mechanism, $g g \rightarrow \Phi$, which dominantly proceeds through $b$-quark triangular loops [6,7] and bottom-quark fusion, $b \bar{b} \rightarrow \Phi$ [8 10], in which the bottom quarks are directly taken from the protons in a five active flavor scheme [11]. The latter process is similar to the channel $p \bar{p} \rightarrow b \bar{b} \Phi$ when no $b$-quarks are detected in the final state [11].

With its successful operation in the last years, the Fermilab Tevatron collider has now collected a substantial amount of data which allows the CDF and D0 experiments to be sensitive to the MSSM Higgs sector. Stringent constraints beyond the well established LEP bounds $M_{A}, M_{h} \gtrsim M_{Z}$ and $\tan \beta \gtrsim 3$ [12], have been set on the MSSM parameter space $\left[M_{A}, \tan \beta\right]$ using the process $g g, b \bar{b} \rightarrow \Phi \rightarrow \tau^{+} \tau^{-}$. Moderate $A$ masses, $M_{A} \approx 100-200 \mathrm{GeV}$, together with high $\tan \beta$ values, $\tan \beta \gtrsim 30$, have been excluded at the $95 \%$ confidence level (CL) [13, 14].

Nevertheless, a very important issue has been overlooked in these experimental analyses: the theoretical uncertainties that affect the production and decay rates, which can be important despite of the fact that some higher order perturbative corrections to these processes are known. These are mainly due to the unknown higher order corrections in perturbation theory, the still not satisfactory parametrization of the parton distribution functions (PDFs), as well as the parametric uncertainties stemming from the not very precisely measured values of the strong coupling constant $\alpha_{s}$ and the bottom quark mass $M_{b}$. In a recent analysis [15], it has been 
shown that these uncertainties can be rather large at the early stage of the CERN large Hadron Collider (lHC), in much the same way as in the case of SM Higgs production both at the IHC [15] and at the Tevatron [16].

In this Letter, we first update the decay branching ratios and production cross sections of the $\Phi$ bosons and find that the latter are significantly lower at high masses than the ones assumed in the Tevatron analyses. We then evaluate the theoretical uncertainties that affect these rates and find them to be very large, possibly lowering the cross sections times branching ratios by a factor $\approx 2$. When included in the D0 and CDF combined analysis of the MSSM Higgs bosons [14, the correct normalization and the uncertainties will drastically reduce the $\operatorname{MSSM}\left[M_{A}, \tan \beta\right]$ parameter space that has been excluded.

For the evaluation of the cross sections in the $g g \rightarrow \Phi$ and $b \bar{b} \rightarrow \Phi$ production processes at the Tevatron, we will concentrate on the pseudoscalar $A$ case and follow very closely the recent analysis performed in Ref. [15] for the lHC. We calculate $\sigma(g g \rightarrow A)$, known up next-to-leading order (NLO) only [7], using the program HIGLU [17] with central values for the renormalization and factorization scales, $\mu_{R}=\mu_{F}=\mu_{0}=\frac{1}{2} M_{A}$; only the dominant loop contribution of the bottom quark is taken into account. For the $b \bar{b} \rightarrow A$ process, known up to next-to-next-toleading order (NNLO) [10], we use the program bbh@nnld 1 with a central scale $\mu_{R}=\mu_{F}=$ $\mu_{0}=\frac{1}{4} M_{A}$. In both cases, we work in the $\overline{\mathrm{MS}}$ scheme for the renormalization of the $b$-quark mass; however, while $\bar{m}_{b}\left(\bar{m}_{b}\right)$ is used in the $g g$ process, $\bar{m}_{b}\left(\mu_{R}\right)$ is adopted in the $b \bar{b}$ channel. The resulting partonic cross sections are then folded with the latest MSTW sets of PDFs [18], consistently at the respective orders, NLO or NNLO, in perturbation theory.

In both the $g g \rightarrow A$ and $b \bar{b} \rightarrow A$ processes, we assume the $b \bar{b} A$ coupling to be SM-like, $\lambda_{A b b}=m_{b} / v$. To obtain the true cross sections, one has to rescale the numbers which will be given by a factor $\tan ^{2} \beta$. In addition, to obtain the cross section for both the $A$ and $H(h)$ bosons, an additional factor of two has to be included. As a consequence of chiral symmetry for $M_{\Phi} \gg \bar{m}_{b}$ and since the $H(h)$ masses and couplings are very close to those of $A$, this represents an excellent approximation 2 .

The results for the cross sections $\sigma(g g \rightarrow A)$ and $\sigma(b \bar{b} \rightarrow A)$ are shown in the main frames of Fig. 1 for the Higgs mass range that is relevant at the Tevatron, $M_{A}=90-200 \mathrm{GeV}$. We have compared our values with those given by the program that has been used by the CDF and D0 collaborations for their cross section normalization, FeynHiggs [22]. This program, initially supposed to only provide precise values for the MSSM Higgs masses and couplings, gives also grids for production cross sections which should be used with care. For the $b \bar{b} \rightarrow A$ channel, we obtain cross sections that are $\approx 30 \%$ smaller. The reason is that FeynHiggs simply provides the values given in the original paper [10] which uses the outdated MRST2002 set of PDFs which are only partly at NNLO. In the case of $g g \rightarrow A$, the agreement is better as we obtain

\footnotetext{
${ }^{1}$ We thank R. Harlander for providing us with his code.

${ }^{2}$ Note that there are additional SUSY contributions in $g g \rightarrow H / h$ which do not appear in $g g \rightarrow A$ but they are very small for a large SUSY breaking scale, $M_{\Phi} \ll M_{S}$ [19. Furthermore, there are one-loop vertex corrections to the $\Phi b \bar{b}$ coupling due to SUSY particles which can be significant as they grow with $\tan \beta[20$. They are implemented in the major codes which calculate the MSSM Higgs spectra and can be readily included. However, in the case of $p \bar{p} \rightarrow \Phi \rightarrow \tau^{+} \tau^{-}$, they almost entirely cancel in the cross section times branching ratios and the remaining part is so small that it has no practical impact whatever benchmark scenario is considered. This can be seen from the almost identical tables XI-XIV and Figs. 4 of Ref. 14 that describe four benchmark scenarios [21].
} 
a cross section that is only $\approx 10 \%$ higher; this can be attributed to the different central scale and renormalization scheme for $M_{b}$ that have been used 3 .
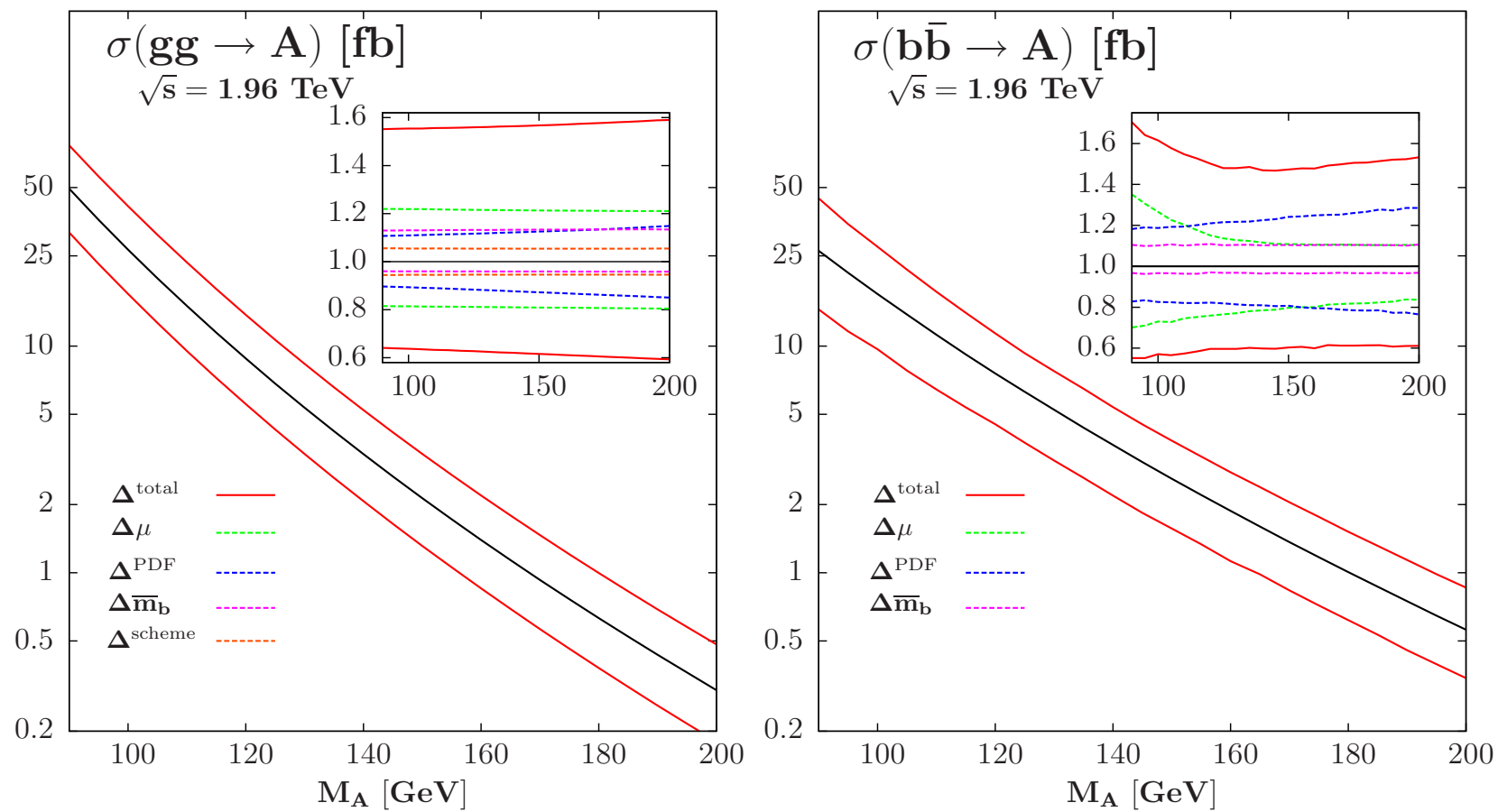

Figure 1: The normalization of the cross sections $\sigma_{g g \rightarrow A}^{\mathrm{NLO}}$ (left) and $\sigma_{b \bar{b} \rightarrow A}^{\mathrm{NNLO}}$ (right) at Tevatron energies as a function of $M_{A}$ when using the MSTW PDFs and unit $A b \bar{b}$ couplings. In the inserts, shown are the various sources of theoretical uncertainties when the rates are normalized to the central values.

For the evaluation of the theoretical uncertainties that affect the Higgs production cross sections as well as the decay branching ratios, we will proceed as follows.

The Higgs decays branching ratios (BR) have been discussed in Ref. [15] and are simply affected by the parametric uncertainties on the input $b$-quark mass and $\alpha_{s}$. As the QCD corrections to the dominant $\Phi \rightarrow b \bar{b}$ decays are large, they are resummed by switching from the $b$-quark pole mass $M_{b}$ which appears at tree-level to the running quark mass in the $\overline{\mathrm{MS}}$ scheme evaluated at the scale of the Higgs mass, $\bar{m}_{b}\left(M_{\Phi}\right)$. The uncertainties come then from: $i)$ the starting point for the $b$-quark mass $\bar{m}_{b}\left(\bar{m}_{b}\right)=4.19_{-0.06}^{+0.18} \mathrm{GeV}$ [23] where the central value corresponds to a pole mass of $M_{b}=4.71 \mathrm{GeV}$; and $\left.i i\right)$ the error on the QCD coupling $\alpha_{s}\left(M_{Z}^{2}\right)=0.1171 \pm 0.0014$ at NNLO (the value adopted in the cross sections) [18] which is used to run the $b$-quark mass from $\bar{m}_{b}$ up to $M_{\Phi}$. Assuming that there is no uncertainty in the $\Phi \rightarrow \tau^{+} \tau^{-}$decay as only electroweak effects are present, and adding the errors in quadrature, one finds an uncertainty of $\approx+4 \%,-9 \%$ on $\operatorname{BR}\left(\Phi \rightarrow \tau^{+} \tau^{-}\right)$and $\approx+1 \%,-0.5 \%$ on $\operatorname{BR}(\Phi \rightarrow b \bar{b})$ at the $1 \sigma$ level over the entire relevant Higgs mass range, $M_{A}=90-200 \mathrm{GeV}$.

In the case of the production cross sections, the uncertainty from the missing higher orders in perturbation theory is usually estimated by varying the renormalization and factorization scales in the domains $\mu_{0} / \kappa \leq \mu_{R}, \mu_{F} \leq \kappa \mu_{0}$ around the central scales $\mu_{0}$, with the additional

\footnotetext{
${ }^{3}$ We thank S. Heinemeyer for a discussion on these points.
} 
restriction $1 / \kappa \leq \mu_{R} / \mu_{F} \leq \kappa$ imposed. While we choose $\kappa=2$ for the $g g \rightarrow A$ process, the value $\kappa=3$ is adopted for $b \bar{b} \rightarrow A$. The reason is that it is well known that the cross sections in $b \bar{b} \rightarrow A$ and in the twin process $q \bar{q}, g g \rightarrow b \bar{b} A$ in a four-flavor scheme differ significantly [24] and by allowing for a wider domain for scale variation and, hence, a larger scale uncertainty, the two results become more consistent with each other. Furthermore, in the $g g \rightarrow A$ process, there is an additional uncertainty that we will consider: the one due to the choice of the scheme for the renormalization of the $b$-quark mass. The latter is estimated by taking the difference between the results obtained in the on-shell mass and $\overline{\mathrm{MS}}$ schemes and allowing for both signs. The inclusion of this additional effect is similar to increasing the domain of scale variation from $\kappa=2$ to $\kappa=3$.

For the combined uncertainties from the PDFs and the QCD coupling $\alpha_{s}$, we will use the scheme made available by the MSTW collaboration [18]. The PDF $+\Delta^{\exp } \alpha_{s}$ uncertainty, with $\alpha_{s}\left(M_{Z}^{2}\right)=0.120 \pm 0.002$ at NLO for $g g \rightarrow A$ and $\alpha_{s}\left(M_{Z}^{2}\right)=0.1171 \pm 0.0014$ at NNLO for $b \bar{b} \rightarrow A$, is evaluated at the $90 \%$ CL. To this, we add in quadrature the impact of a theoretical error on $\alpha_{s}$, estimated by MSTW to be $\Delta^{\text {th }} \alpha_{s} \approx 0.003$ at NLO and $\Delta^{\text {th }} \approx 0.002$ at NNLO. Finally, in the case of the $b \bar{b} \rightarrow A$ process, there is an effect induced by the uncertainty in the value of $M_{b}$ in the $b$-quark density. This effect is again estimated within the MSTW scheme by allowing for an uncertainty on the pole $b$-mass of $\pm 0.25 \mathrm{GeV}$ from the MSTW central value $M_{b}=4.75 \pm 0.25 \mathrm{GeV}$. This resulting uncertainty is also added in quadrature to the $\mathrm{PDF}+\Delta^{\exp } \alpha_{s}$ one. Note that we have evaluated both cross sections with four other PDF sets and found that the maximal values are obtained with MSTW in $g g \rightarrow A$, while some other schemes give $\approx 20 \%$ lower rates.

Finally, there is the uncertainty on the $b$-quark mass that affects the amplitudes of these processes, in much the same way as what has been discussed for the Higgs decay branching ratios. It is estimated by evaluating the maximal values of the cross sections when one includes the error on the input $\overline{\mathrm{MS}} b$-quark mass at the scale $\bar{m}_{b}, \bar{m}_{b}\left(\bar{m}_{b}\right)=4.19_{-0.06}^{+0.18} \mathrm{GeV}$, and in the case of the $b \bar{b} \rightarrow A$ process where the Yukawa coupling is defined at the high scale, $\propto \bar{m}_{b}\left(\mu_{R}\right)$, the error on the coupling $\alpha_{s}\left(M_{Z}^{2}\right)=0.1171 \pm 0.0014$ at NNLO (in this case, it is almost the same uncertainty as in the $A \rightarrow b \bar{b}$ decay).

The results for these uncertainties on the production cross sections at the Tevatron are shown in Fig. 1 for the $g g \rightarrow A$ and $b \bar{b} \rightarrow A$ processes as a function of $M_{A}$. In the $g g$ case and almost independently of $M_{A}$, the scale variation in a domain with $\kappa=2$ leads to an uncertainty $\mathcal{O}( \pm 20 \%)$, while the uncertainty from the scheme dependence in the renormalization of $M_{b}$ is about $\pm 6 \%$; they add up to $\approx 25 \%$ that is only slightly lower than the scale uncertainty in the $b \bar{b}$ process, $\approx 30 \%$ for low $M_{A}$, in which the domain of variation is extended to $\kappa=3$. In the $g g \rightarrow A(b \bar{b} \rightarrow A)$ channel, the $\mathrm{PDF}+\Delta^{\exp +\text { th }} \alpha_{s}$ (with $\Delta M_{b}$ in addition for $b \bar{b} \rightarrow A$ ) uncertainties are at the level of $\pm 10 \%( \pm 20 \%)$ for $M_{A} \approx 100 \mathrm{GeV}$ and larger $( \pm 30 \%$ in $b \bar{b} \rightarrow A)$ at $M_{A} \approx 200 \mathrm{GeV}$ where the more uncertain high Bjorken- $x$ values for the gluon and bottom quark densities are probed. The parametric error on $\bar{m}_{b}$ leads to a $\approx+13 \%,-4 \%$ uncertainty in the $g g \rightarrow A$ process and slightly less in the case of $b \bar{b} \rightarrow A$.

We turn now to the issue of combining these uncertainties. Clearly, the scale and scheme uncertainties, which are purely theoretical and both emerge from the truncation of the perturbative series, should be added linearly. The PDF $+\Delta \alpha_{s}+\Delta m_{b}$ uncertainty, that we also would like to view as a reflection of the theoretical ambiguities due to the parametrization of the 
PDFs and which have no statistical ground, will be evaluated on the minimal and maximal values of the cross sections with respect to scale and scheme variation (see Ref. [15] for the argumentation). This procedure gives results that are similar to those obtained with a linear addition of the scale+scheme and $\mathrm{PDF}+\Delta \alpha_{s}+\Delta m_{b}$ uncertainties as advocated in, for instance, Ref. [25]. Finally, at this stage, we add linearly the parametric uncertainty on $\bar{m}_{b}$ which, in the case of interest, will drop anyway in the final result (see below).

The combined uncertainties on the cross sections, when using this procedure, are also shown in Fig. 1 for the two production channels. As can be seen, they are very large: at low Higgs masses, $M_{A} \approx 100 \mathrm{GeV}$, one has $\approx+55 \%,-35 \%$ for $\sigma(g g \rightarrow A)$ and $\approx+60 \%,-40 \%$ for $\sigma(b \bar{b} \rightarrow A)$ which become at masses $M_{A} \approx 200 \mathrm{GeV}$, respectively, $\approx+60 \%,-40 \%$ and $\approx+50 \%,-40 \%$.

To consider the final state topology that has been searched for by the D0 and CDF collaborations [14, i.e. $p \bar{p} \rightarrow$ Higgs $\rightarrow \tau^{+} \tau^{-}$, one has first to add the cross sections for the two channels $g g \rightarrow A$ and $b \bar{b} \rightarrow A$, and then to multiply the resulting production cross section by the Higgs branching ratio $\operatorname{BR}\left(A \rightarrow \tau^{+} \tau^{-}\right) \approx 10 \%$. The resulting $\sigma(p \bar{p} \rightarrow A) \times \operatorname{BR}\left(A \rightarrow \tau^{+} \tau^{-}\right)$ at the Tevatron is shown in Fig. 2 as a function of $M_{A}$. We stress again that to obtain the true rate for $\Phi=A+H(h)$, one has to multiply the given values by a factor $2 \tan ^{2} \beta$.

In Fig. 2, shown also are the associated overall theoretical uncertainties. The uncertainty from the cross section alone is dominated by that of $g g \rightarrow A$ at low Higgs masses and $b \bar{b} \rightarrow A$ at high masses as the corresponding cross sections are largest. In the product $\sigma(p \bar{p} \rightarrow A) \times$ $\mathrm{BR}\left(A \rightarrow \tau^{+} \tau^{-}\right)$, the parametric uncertainty that its common to the production and decay rates almost cancels out as shown by the solid curves in Fig. 2 and only a few percent are left. This leads to a smaller uncertainty in $\sigma(p \bar{p} \rightarrow A) \times \operatorname{BR}\left(A \rightarrow \tau^{+} \tau^{-}\right)$than in $\sigma(p \bar{p} \rightarrow A)$ alone. The final theoretical uncertainty for $p \bar{p} \rightarrow A \rightarrow \tau^{+} \tau^{-}$at the Tevatron is of order $+50 \%,-40 \%$.

To illustrate the impact of these theoretical uncertainties on the MSSM $\left[M_{A}, \tan \beta\right]$ parameter space that is probed when searching experimentally for the $p \bar{p} \rightarrow \Phi \rightarrow \tau^{+} \tau^{-}$channel, we show in Fig. 3 the contour of the cross section times branching ratio in this plane, together with the contours when the uncertainties are included. We apply the model independent 95\%CL expected and observed limits from the CDF/D0 analysis (Table X of Ref. [14]). However, rather than applying the limits on the central $\sigma \times \mathrm{BR}$ rate, we apply them on the minimal one when the theory uncertainty is included. Indeed, since the latter has a flat prior, the minimal $\sigma \times \mathrm{BR}$ value is as respectable and likely as the central value. One observes then that only values $\tan \beta \gtrsim 50$ are excluded in the mass ranges, $M_{\Phi} \approx 95-125 \mathrm{GeV}$ and $M_{\Phi} \gtrsim 165 \mathrm{GeV}$. In the intermediate range $M_{\Phi} \approx 125-165 \mathrm{GeV}$, the exclusion limit is $\tan \beta \gtrsim 40-45$, to be contrasted with the values $\tan \beta \gtrsim 30$ excluded in the CDF/D0 analysis. Hence, the inclusion of the theory uncertainties has a drastic impact on the allowed $\left[M_{A}, \tan \beta\right]$ parameter space.

Finally, let us note that there is a subleading channel which has also been considered at the Tevatron, $b g \rightarrow \Phi b \rightarrow 3 b$ [26]. The evaluation of the theory uncertainties in $b g \rightarrow \Phi b[9]$ follows that of the parent process $b \bar{b} \rightarrow \Phi$ (for which it is part of the NLO contributions) and similar results, i.e. a total uncertainty of $\approx \pm 40 \%$, are expected. However, in this case, it is the $\Phi \rightarrow b \bar{b}$ decay which is considered experimentally and, since $\operatorname{BR}(\Phi \rightarrow b \bar{b})$ has a small error, the uncertainties in $\sigma$ and $\sigma \times \mathrm{BR}$ are almost the same. These uncertainties will thus also impact the excluded $\left[M_{A}, \tan \beta\right]$ parameter space 4 .

\footnotetext{
${ }^{4}$ Note that the $2 \sigma$ excess observed by CDF in this channel cannot be a Higgs signal as it would correspond
} 


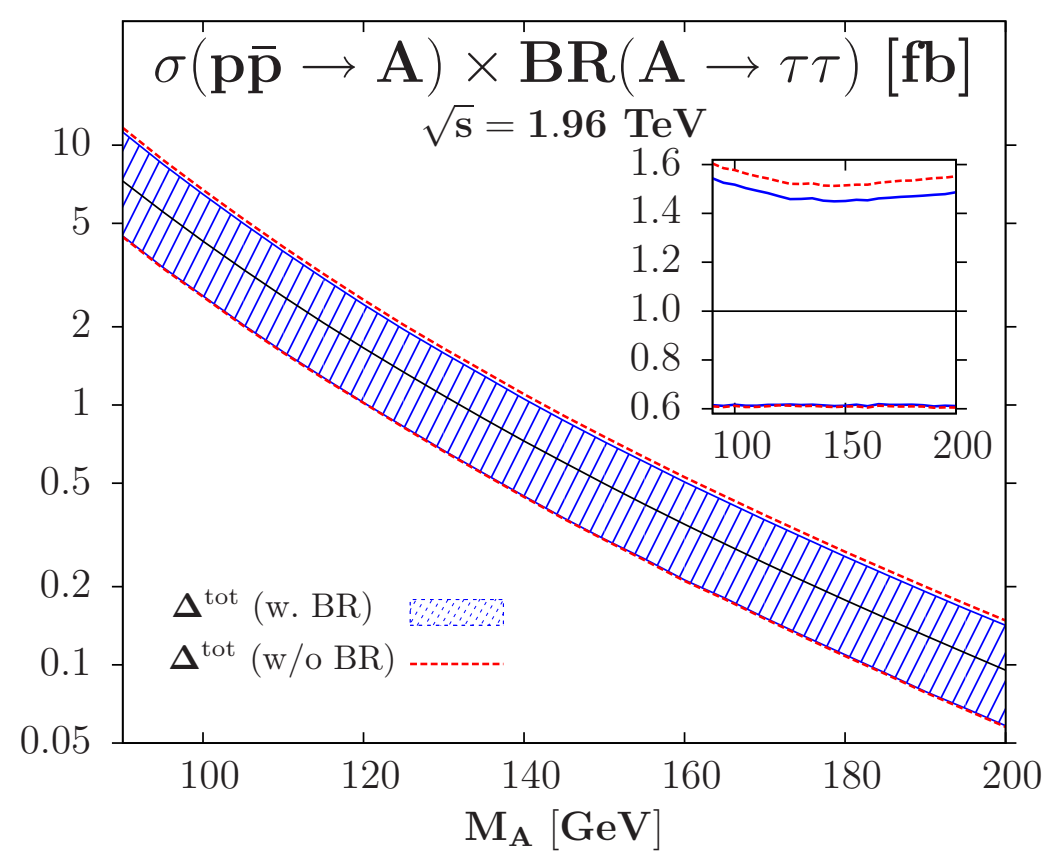

Figure 2: $\sigma(p \bar{p} \rightarrow A) \times \mathrm{BR}\left(A \rightarrow \tau^{+} \tau^{-}\right)$as a function of $M_{A}$ at the Tevatron, together with the associated overall theoretical uncertainty; the uncertainty when excluding that on the branching ratio is also displayed. In the inserts, shown are the relative deviations from the central values.

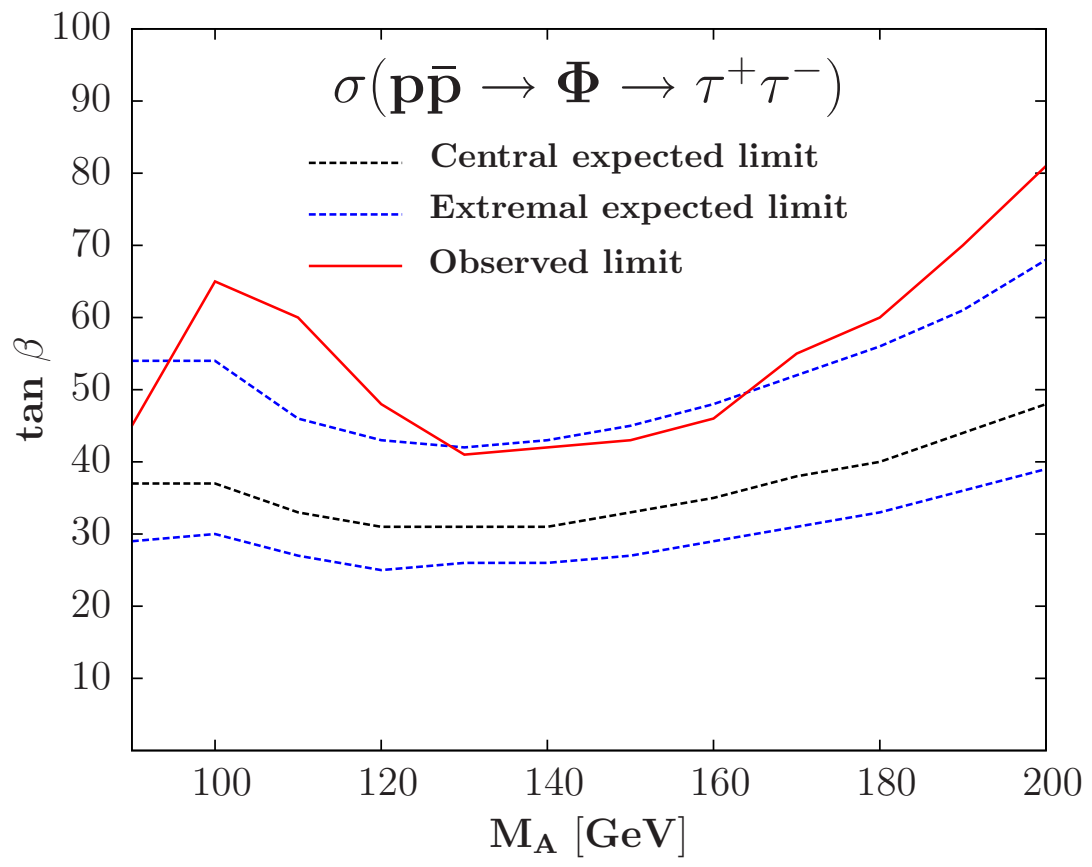

Figure 3: Contours for the expected $\sigma\left(p \bar{p} \rightarrow \Phi \rightarrow \tau^{+} \tau^{-}\right)$rate at the Tevatron in the $\left[M_{A}, \tan \beta\right]$ plane with the associated theory uncertainties, confronted to the $95 \%$ CL exclusion limit.

to a much larger excess in $p \bar{p} \rightarrow \tau^{+} \tau^{-}$which has not been observed. In addition, this $3 b$ channel probes

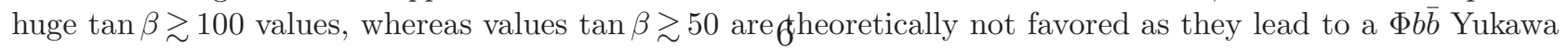
coupling that is non perturbative. For such values, the total Higgs widths are to be included. 
In conclusion, we have updated the cross sections for the production of the MSSM CP-odd like Higgs bosons $\Phi$ at the Tevatron in the processes $g g \rightarrow \Phi$ and $b \bar{b} \rightarrow \Phi$ and found smaller rates in the high Higgs mass range compared to those assumed by the CDF and D0 experiments. We have then evaluated the associated theoretical uncertainties, including also the ones in the $\Phi \rightarrow \tau^{+} \tau^{-}$decay branching fractions, and find that they are very large. These uncertainties, together with the correct normalization, affect significantly the exclusion limits set on the MSSM parameter space from the negative Higgs searches in the channel $p \bar{p} \rightarrow \Phi \rightarrow \tau^{+} \tau^{-}$at the Tevatron. Additional material is given in Tables 1 and 2 which summarize our results.

Acknowledgments: Discussions with Michael Spira are gratefully acknowledged. This work is supported by the European network HEPTOOLS.

\section{References}

[1] P. Higgs, Phys. Lett. 12 (1964) 132; F. Englert and R. Brout, Phys. Rev. Lett. 13 (1964) 321.

[2] For a review see, M. Drees, R. Godbole and P. Roy, Theory and Phenomenology of Sparticles, World Sci, 2004.

[3] J. Gunion, H. Haber, G. Kane and S. Dawson, The Higgs Hunter's Guide, AddisonWesley, Reading 1990.

[4] For more recent reviews, see: A. Djouadi, Phys. Rept. 457 (2008) 1; Phys. Rep. 459 (2008) 1.

[5] A. Djouadi, J. Kalinowski and M. Spira, Comput. Phys. Commun. 108 (1998) 56.

[6] H. Georgi et al., Phys. Rev. Lett. 40 (1978) 692.

[7] M. Spira et al., Nucl. Phys. B453 (1995) 17.

[8] D. Dicus and S. Willenbrock, Phys. Rev. D39 (1989) 751.

[9] J. Campbell et al., Phys. Rev. D67 (2003) 095002; F. Maltoni, Z. Sullivan and S. Willenbrock Phys. Rev. D67 (2003) 093005.

[10] R. Harlander, W. Kilgore, Phys. Rev. D68 (2003) 013001.

[11] S. Dittmaier, M. Kramer and M. Spira, Phys. Rev. D70 (2004) 074010; S. Dawson et al., Phys. Rev. D69 (2004) 074027.

[12] The LEP collaborations, Phys. Lett. B565 (2003) 61.

[13] CDF collaboration, Phys. Rev. Lett. 103 (2009) 201801; D0 collaboration, Phys. Rev. Lett. 101 (2008) 071804.

[14] The CDF/D0 TevNPHWG, arXiv:1003.3363 [hep-ex]. 
[15] J. Baglio and A. Djouadi, JHEP 1103 (2011) 055.

[16] J. Baglio and A. Djouadi, JHEP 1010 (2010) 064.

[17] M. Spira, Fortschr. Phys. 46 (1998) 203; hep-ph/9510347.

[18] A.D. Martin, W. Stirling, R. Thorne and G. Watt, Eur. Phys. J. C63 (2009) 189; Eur. Phys. J. C64 (2009) 653; arXiv:1007.2624 [hep-ph].

[19] See e.g., M. Muhlleitner, H. Rzehak and M. Spira, arXiv:101.3214 [hep-ph] and references therein.

[20] See e.g., M. Carena et al., Nucl. Phys. B577 (2000) 88; D. Noth and M. Spira, Phys. Rev. Lett. 101 (2008) 181801.

[21] M. Carena et al., Eur. J. Phys. C26 (2003) 601.

[22] S. Heinemeyer, W. Hollik and G. Weiglein, Comp. Phys. Commun. 124 (2000) 76.

[23] K. Nakamura et al., J. Phys. G37 (2010) 075021.

[24] J. Campbell et al., hep-ph/0406152.

[25] S. Dittmaier et al., LHC Higgs cross section Working Group, arXiv:1101.0593.

[26] CDF collaboration, note CDF/10105 (June 2010). 


\begin{tabular}{|c|c|c|c|c|c|c|c|c|c|c|}
\hline$M_{A}$ & $\sigma_{\mathrm{gg} \rightarrow \mathrm{A}}^{\mathrm{NLO}}$ & scale & scheme & PDFs & param & total & \multicolumn{2}{|c|}{$\operatorname{BR}(\tau \tau)$} & \multicolumn{2}{|c|}{$\sigma \times \mathrm{BR}$} \\
\hline 90 & 49.46 & $\begin{array}{l}+22.0 \% \\
-18.5 \% \\
\end{array}$ & $\begin{array}{l}+5.6 \% \\
-5.6 \% \\
\end{array}$ & $\begin{array}{l}+10.7 \% \\
-10.3 \% \\
\end{array}$ & $\begin{array}{l}+13.0 \% \\
-4.0 \% \\
\end{array}$ & $\begin{array}{l}+55.2 \% \\
-35.9 \% \\
\end{array}$ & 9.60 & $\begin{array}{l}3.8 \% \\
-8.8 \% \\
\end{array}$ & 4.75 & $\begin{array}{l}+49.7 \% \\
-35.7 \% \\
\end{array}$ \\
\hline 95 & 36.07 & $\begin{array}{l}+22.0 \% \\
-18.6 \%\end{array}$ & $\begin{array}{l}+5.6 \% \\
-5.6 \% \\
\end{array}$ & $\begin{array}{l}+10.8 \% \\
-10.5 \%\end{array}$ & $\begin{array}{l}+13.0 \% \\
-4.0 \%\end{array}$ & $\begin{array}{l}+55.3 \% \\
-36.1 \% \\
\end{array}$ & 9.70 & $\begin{array}{l}3.7 \% \\
-8.8 \% \\
\end{array}$ & 3.50 & $\begin{array}{l}+49.9 \% \\
-35.9 \% \\
\end{array}$ \\
\hline 100 & 26.62 & $\begin{array}{l}+21.9 \% \\
-18.6 \% \\
\end{array}$ & $\begin{array}{l}+5.5 \% \\
-5.5 \% \\
\end{array}$ & $\begin{array}{l}+10.9 \% \\
-10.7 \%\end{array}$ & $\begin{array}{l}+13.1 \% \\
-4.0 \%\end{array}$ & $\begin{array}{l}+55.4 \% \\
-36.3 \% \\
\end{array}$ & 9.79 & $\begin{array}{r}+3.8 \% \\
-8.8 \% \\
\end{array}$ & 2.61 & $\begin{array}{l}+50.0 \% \\
-36.0 \% \\
\end{array}$ \\
\hline 105 & 19.90 & $\begin{array}{l}+21.9 \% \\
-18.7 \% \\
\end{array}$ & $\begin{array}{l}+5.5 \% \\
-5.5 \% \\
\end{array}$ & $\begin{array}{l}11.1 \% \\
-10.8 \% \\
\end{array}$ & $\begin{array}{l}+13.0 \% \\
-4.0 \%\end{array}$ & $\begin{array}{l}+55.5 \% \\
-36.5 \% \\
\end{array}$ & 9.88 & $\begin{array}{l}3.8 \% \\
-8.8 \% \\
\end{array}$ & 1.97 & $\begin{array}{l}+50.1 \% \\
-36.3 \% \\
\end{array}$ \\
\hline 110 & 15.03 & $\begin{array}{l}+21.8 \% \\
-18.7 \% \\
\end{array}$ & $\begin{array}{l}+5.5 \% \\
-5.5 \% \\
\end{array}$ & $\begin{array}{l}+11.2 \% \\
-11.0 \%\end{array}$ & $\begin{array}{l}+13.1 \% \\
-4.1 \%\end{array}$ & $\begin{array}{l}+55.7 \% \\
-36.7 \% \\
\end{array}$ & 9.96 & $\begin{array}{l}+3.8 \% \\
-8.8 \% \\
\end{array}$ & 1.50 & $\begin{array}{l}+50.3 \% \\
-36.6 \% \\
\end{array}$ \\
\hline 115 & 11.46 & $\begin{array}{l}+21.7 \% \\
-18.8 \%\end{array}$ & $\begin{array}{l}+5.5 \% \\
-5.5 \%\end{array}$ & $\begin{array}{l}+11.4 \% \\
-11.2 \%\end{array}$ & $\begin{array}{l}+13.1 \% \\
-4.0 \%\end{array}$ & $\begin{array}{l}+55.7 \% \\
-36.9 \%\end{array}$ & 10.04 & $\begin{array}{l}+3.8 \% \\
-8.8 \%\end{array}$ & 1.15 & $\begin{array}{l}+50.4 \% \\
-36.7 \%\end{array}$ \\
\hline 120 & 8.82 & $\begin{array}{l}+21.6 \% \\
-18.8 \% \\
\end{array}$ & $\begin{array}{l}+5.5 \% \\
-5.5 \% \\
\end{array}$ & $\begin{array}{l}11.5 \% \\
-11.4 \% \\
\end{array}$ & $\begin{array}{l}+13.2 \% \\
-4.1 \% \\
\end{array}$ & $\begin{array}{l}+55.8 \% \\
-37.1 \% \\
\end{array}$ & 10.12 & $\begin{array}{l}+3.8 \% \\
-8.8 \% \\
\end{array}$ & 0.89 & $\begin{array}{l}+50.5 \% \\
-37.0 \% \\
\end{array}$ \\
\hline 125 & 6.84 & $\begin{array}{l}+21.6 \% \\
-18.8 \% \\
\end{array}$ & $\begin{array}{l}+5.4 \% \\
-5.4 \% \\
\end{array}$ & $\begin{array}{l}+11.7 \% \\
-11.7 \% \\
\end{array}$ & $\begin{array}{l}+13.2 \% \\
-4.1 \% \\
\end{array}$ & $\begin{array}{r}+56.0 \% \\
-37.3 \%\end{array}$ & 10.19 & $\begin{array}{l}+3.0 \% \\
-8.8 \%\end{array}$ & 0.70 & $\begin{array}{r}+50.6 \% \\
-37.1 \%\end{array}$ \\
\hline 130 & 5.35 & $\begin{array}{l}+21.5 \% \\
-18.9 \%\end{array}$ & $\begin{array}{l}+5.4 \% \\
-5.4 \%\end{array}$ & $\begin{array}{l}+11.8 \% \\
-11.9 \%\end{array}$ & $\begin{array}{l}+13.2 \% \\
-4.1 \%\end{array}$ & $\begin{array}{l}+56.0 \% \\
-37.6 \%\end{array}$ & 10.26 & $\begin{array}{l}+3.8 \% \\
-8.7 \%\end{array}$ & 0.55 & $\begin{array}{l}+50.9 \% \\
-37.4 \%\end{array}$ \\
\hline 135 & 4.21 & $\begin{array}{l}+21.5 \% \\
-18.9 \% \\
\end{array}$ & $\begin{array}{l}+5.4 \% \\
-5.4 \% \\
\end{array}$ & $\begin{array}{l}+12.0 \% \\
-12.1 \% \\
\end{array}$ & $\begin{array}{l}+13.3 \% \\
-4.1 \% \\
\end{array}$ & $\begin{array}{r}+56.3 \% \\
-37.8 \%\end{array}$ & 10.33 & $\begin{array}{l}+3.8 \% \\
-8.8 \%\end{array}$ & 0.43 & $\begin{array}{r}+51.0 \% \\
-37.8 \%\end{array}$ \\
\hline 140 & 3.34 & $\begin{array}{l}+21.4 \% \\
-19.0 \%\end{array}$ & $\begin{array}{l}+5.4 \% \\
-5.4 \%\end{array}$ & $\begin{array}{l}+12.2 \% \\
-12.3 \%\end{array}$ & $\begin{array}{l}+13.3 \% \\
-4.1 \%\end{array}$ & $\begin{array}{l}+56.4 \% \\
-38.0 \%\end{array}$ & 10.39 & $\begin{array}{l}+3.8 \% \\
-8.7 \%\end{array}$ & 0.35 & $\begin{array}{l}+51.2 \% \\
-37.9 \%\end{array}$ \\
\hline 145 & 2.66 & $\begin{array}{l}+21.4 \% \\
-19.0 \%\end{array}$ & $\begin{array}{l}+5.4 \% \\
-5.4 \%\end{array}$ & $\begin{array}{l}+12.3 \% \\
-12.5 \%\end{array}$ & $\begin{array}{l}+13.3 \% \\
-4.1 \%\end{array}$ & $\begin{array}{l}+56.6 \% \\
-38.2 \%\end{array}$ & 10.46 & $\begin{array}{l}+3.7 \% \\
-8.8 \%\end{array}$ & 0.28 & $\begin{array}{l}+51.3 \% \\
-38.4 \%\end{array}$ \\
\hline 150 & 2.13 & $\begin{array}{l}+21.3 \% \\
-19.1 \%\end{array}$ & $\begin{array}{l}+5.4 \% \\
-5.4 \% \\
\end{array}$ & $\begin{array}{l}+12.5 \% \\
-12.8 \% \\
\end{array}$ & $\begin{array}{l}+13.3 \% \\
-4.1 \%\end{array}$ & $\begin{array}{l}+56.8 \% \\
-38.4 \% \\
\end{array}$ & 10.52 & $\begin{array}{l}+3.7 \% \\
-8.8 \% \\
\end{array}$ & 0.22 & $\begin{array}{l}+51.5 \% \\
-38.6 \% \\
\end{array}$ \\
\hline 155 & 1.72 & $\begin{array}{l}+21.3 \% \\
-19.1 \%\end{array}$ & $\begin{array}{l}+5.4 \% \\
-5.4 \%\end{array}$ & $\begin{array}{l}+12.7 \% \\
-13.0 \%\end{array}$ & $\begin{array}{l}+13.3 \% \\
-4.1 \%\end{array}$ & $\begin{array}{l}+56.9 \% \\
-38.7 \%\end{array}$ & 10.57 & $\begin{array}{l}+3.8 \% \\
-8.7 \% \\
\end{array}$ & 0.18 & $\begin{array}{l}+51.8 \% \\
-38.5 \%\end{array}$ \\
\hline 160 & 1.40 & $\begin{array}{l}+21.2 \% \\
-19.2 \%\end{array}$ & $\begin{array}{l}+5.4 \% \\
-5.4 \%\end{array}$ & $\begin{array}{l}+12.9 \% \\
-13.2 \%\end{array}$ & $\begin{array}{l}+13.4 \% \\
-4.1 \%\end{array}$ & $\begin{array}{l}+57.1 \% \\
-38.9 \%\end{array}$ & 10.63 & $\begin{array}{l}+3.8 \% \\
-8.7 \% \\
\end{array}$ & 0.15 & $\begin{array}{l}+52.0 \% \\
-38.9 \%\end{array}$ \\
\hline 165 & 1.14 & $\begin{array}{l}+21.2 \% \\
-19.2 \%\end{array}$ & $\begin{array}{l}+5.4 \% \\
-5.4 \%\end{array}$ & $\begin{array}{l}+13.1 \% \\
-13.4 \%\end{array}$ & $\begin{array}{l}+13.4 \% \\
-4.1 \%\end{array}$ & $\begin{array}{l}+57.4 \% \\
-39.1 \%\end{array}$ & 10.68 & $\begin{array}{l}+3.9 \% \\
-8.7 \% \\
\end{array}$ & 0.12 & $\begin{array}{l}+52.3 \% \\
-39.1 \%\end{array}$ \\
\hline 170 & 0.93 & $\begin{array}{l}+21.2 \% \\
-19.3 \%\end{array}$ & $\begin{array}{l}+5.4 \% \\
-5.4 \%\end{array}$ & $\begin{array}{l}+13.3 \% \\
-13.6 \%\end{array}$ & $\begin{array}{l}+13.4 \% \\
-4.1 \%\end{array}$ & $\begin{array}{l}+57.6 \% \\
-39.3 \%\end{array}$ & 10.74 & $\begin{array}{r}+3.8 \% \\
-8.8 \% \\
\end{array}$ & 0.10 & $\begin{array}{l}+52.4 \% \\
-39.4 \%\end{array}$ \\
\hline 175 & 0.76 & $\begin{array}{l}+21.2 \% \\
-19.3 \%\end{array}$ & $\begin{array}{l}+5.4 \% \\
-5.4 \%\end{array}$ & $\begin{array}{l}+13.5 \% \\
-13.9 \%\end{array}$ & $\begin{array}{l}+13.4 \% \\
-4.2 \%\end{array}$ & $\begin{array}{l}+57.8 \% \\
-39.6 \%\end{array}$ & 10.79 & $\begin{array}{l}+3.8 \% \\
-8.7 \% \\
\end{array}$ & 0.08 & $\begin{array}{l}+52.7 \% \\
-39.5 \%\end{array}$ \\
\hline 180 & 0.63 & $\begin{array}{l}+21.1 \% \\
-19.4 \%\end{array}$ & $\begin{array}{l}+5.4 \% \\
-5.4 \%\end{array}$ & $\begin{array}{l}+13.7 \% \\
-14.1 \%\end{array}$ & $\begin{array}{l}+13.4 \% \\
-4.1 \%\end{array}$ & $\begin{array}{l}+58.1 \% \\
-39.8 \%\end{array}$ & 10.84 & $\begin{array}{l}+3.9 \% \\
-8.7 \% \\
\end{array}$ & 0.07 & $\begin{array}{l}+53.1 \% \\
-39.7 \%\end{array}$ \\
\hline 185 & 0.52 & $\begin{array}{l}+21.1 \% \\
-19.4 \%\end{array}$ & $\begin{array}{l}+5.4 \% \\
-5.4 \%\end{array}$ & $\begin{array}{l}+14.0 \% \\
-14.3 \%\end{array}$ & $\begin{array}{l}+13.4 \% \\
-4.2 \%\end{array}$ & $\begin{array}{l}+58.3 \% \\
-40.0 \% \\
\end{array}$ & 10.90 & $\begin{array}{l}+3.7 \% \\
-8.8 \% \\
\end{array}$ & 0.06 & $\begin{array}{l}+53.1 \% \\
-40.2 \%\end{array}$ \\
\hline 190 & 0.43 & $\begin{array}{l}+21.1 \% \\
-19.5 \%\end{array}$ & $\begin{array}{l}+5.4 \% \\
-5.4 \%\end{array}$ & $\begin{array}{l}+14.3 \% \\
-14.5 \%\end{array}$ & $\begin{array}{l}+13.5 \% \\
-4.2 \%\end{array}$ & $\begin{array}{l}+58.5 \% \\
-40.3 \%\end{array}$ & 10.95 & $\begin{array}{r}+3.8 \% \\
-8.7 \% \\
\end{array}$ & 0.05 & $\begin{array}{l}+53.3 \% \\
-40.4 \%\end{array}$ \\
\hline 195 & 0.36 & $\begin{array}{l}+21.1 \% \\
-19.5 \%\end{array}$ & $\begin{array}{l}+5.4 \% \\
-5.4 \%\end{array}$ & $\begin{array}{l}+14.6 \% \\
-14.7 \%\end{array}$ & $\begin{array}{l}+13.4 \% \\
-4.2 \%\end{array}$ & $\begin{array}{l}+58.8 \% \\
-40.5 \% \\
\end{array}$ & 11.00 & $\begin{array}{l}+3.8 \% \\
-8.8 \% \\
\end{array}$ & 0.04 & $\begin{array}{l}+53.7 \% \\
-40.6 \%\end{array}$ \\
\hline 200 & 0.30 & $\begin{array}{l}+21.1 \% \\
-19.6 \%\end{array}$ & $\begin{array}{l}+5.4 \% \\
-5.4 \%\end{array}$ & $\begin{array}{l}+14.9 \% \\
-15.0 \%\end{array}$ & $\begin{array}{l}+13.4 \% \\
-4.2 \%\end{array}$ & $\begin{array}{l}+59.0 \% \\
-40.7 \%\end{array}$ & 11.04 & $\begin{array}{l}+3.9 \% \\
-8.7 \% \\
\end{array}$ & 0.03 & $\begin{array}{l}+54.2 \% \\
-40.7 \%\end{array}$ \\
\hline
\end{tabular}

Table 1: The production cross sections in the $g g \rightarrow A$ process at the Tevatron (in fb) for given $A$ masses (in $\mathrm{GeV}$ ) at a scale $\mu_{F}=\mu_{R}=\frac{1}{2} M_{A}$ with MSTW PDFs. Shown also are the corresponding uncertainties from the various sources discussed as well as the total uncertainty. In the other columns, displayed are the branching ratio $\mathrm{BR}\left(A \rightarrow \tau^{+} \tau^{-}\right)$[in \%] and the product $\sigma(g g \rightarrow A) \times \mathrm{BR}\left(A \rightarrow \tau^{+} \tau^{-}\right)$together with their respective total uncertainties. 


\begin{tabular}{|c|c|c|c|c|c|c|c|c|c|}
\hline$M_{A}$ & $\sigma_{\mathrm{b} \overline{\mathrm{b}} \rightarrow \mathrm{A}}^{\mathrm{NNLO}}$ & scale & PDFs & param & total & \multicolumn{2}{|c|}{$\operatorname{BR}(\tau \tau)$} & \multicolumn{2}{|c|}{$\sigma \times \mathrm{BR}$} \\
\hline 90 & 26.31 & $\begin{array}{l}+35.1 \% \\
-29.9 \% \\
\end{array}$ & $\begin{array}{l}+18.2 \% \\
-17.2 \%\end{array}$ & $\begin{array}{l}+10.5 \% \\
-3.3 \%\end{array}$ & $\begin{array}{l}+70.4 \% \\
-44.9 \% \\
\end{array}$ & 9.60 & $\begin{array}{l}+3.8 \% \\
-8.8 \% \\
\end{array}$ & 2.53 & $\begin{array}{l}+63.4 \% \\
-44.0 \%\end{array}$ \\
\hline 95 & 21.04 & $\begin{array}{l}+30.6 \% \\
-29.0 \% \\
\end{array}$ & $\begin{array}{l}19.0 \% \\
-16.6 \% \\
\end{array}$ & $\begin{array}{l}+9.9 \% \\
-3.7 \% \\
\end{array}$ & $\begin{array}{l}+64.1 \% \\
-44.9 \% \\
\end{array}$ & 9.70 & $\begin{array}{l}+3.7 \% \\
-8.8 \% \\
\end{array}$ & 2.04 & $\begin{array}{l}+57.1 \% \\
-43.9 \% \\
\end{array}$ \\
\hline 100 & 16.96 & $\begin{array}{l}+26.6 \% \\
-27.0 \% \\
\end{array}$ & $\begin{array}{l}+18.7 \% \\
-17.5 \% \\
\end{array}$ & $\begin{array}{l}+10.2 \% \\
-3.4 \%\end{array}$ & $\begin{array}{l}+61.5 \% \\
-42.9 \% \\
\end{array}$ & 9.79 & $\begin{array}{l}+3.8 \% \\
-8.8 \% \\
\end{array}$ & 1.66 & $\begin{array}{l}+54.5 \% \\
-42.0 \%\end{array}$ \\
\hline 105 & 13.78 & $\begin{array}{l}+22.6 \% \\
-27.2 \% \\
\end{array}$ & $\begin{array}{l}+19.2 \% \\
-17.6 \% \\
\end{array}$ & $\begin{array}{l}+10.7 \% \\
-3.4 \% \\
\end{array}$ & $\begin{array}{l}+57.8 \% \\
-43.4 \% \\
\end{array}$ & 9.88 & $\begin{array}{l}+3.8 \% \\
-8.8 \% \\
\end{array}$ & 1.36 & $\begin{array}{l}+50.8 \% \\
-42.4 \% \\
\end{array}$ \\
\hline 110 & 11.22 & $\begin{array}{l}+20.3 \% \\
-25.5 \%\end{array}$ & $\begin{array}{l}+19.4 \% \\
-17.9 \%\end{array}$ & $\begin{array}{l}+10.1 \% \\
-3.6 \%\end{array}$ & $\begin{array}{l}+54.7 \% \\
-42.6 \% \\
\end{array}$ & 9.96 & $\begin{array}{l}+3.8 \% \\
-8.8 \% \\
\end{array}$ & 1.12 & $\begin{array}{l}+47.9 \% \\
-41.7 \%\end{array}$ \\
\hline 115 & 9.18 & $\begin{array}{l}+17.6 \% \\
-24.8 \% \\
\end{array}$ & $\begin{array}{l}+20.1 \% \\
-17.9 \% \\
\end{array}$ & $\begin{array}{l}+10.6 \% \\
-3.6 \% \\
\end{array}$ & $\begin{array}{l}+52.7 \% \\
-41.6 \% \\
\end{array}$ & 10.04 & $\begin{array}{l}+3.8 \% \\
-8.8 \% \\
\end{array}$ & 0.92 & $\begin{array}{l}+45.7 \% \\
-40.6 \% \\
\end{array}$ \\
\hline 120 & 7.57 & $\begin{array}{l}+15.1 \% \\
-24.1 \% \\
\end{array}$ & $\begin{array}{l}+21.0 \% \\
-17.8 \% \\
\end{array}$ & $\begin{array}{l}+10.9 \% \\
-3.0 \%\end{array}$ & $\begin{array}{l}+50.2 \% \\
-40.3 \% \\
\end{array}$ & 10.12 & $\begin{array}{l}+3.8 \% \\
-8.8 \% \\
\end{array}$ & 0.77 & $\begin{array}{l}+43.1 \% \\
-40.0 \% \\
\end{array}$ \\
\hline 125 & 6.29 & $\begin{array}{l}+13.6 \% \\
-23.5 \% \\
\end{array}$ & $\begin{array}{l}+21.5 \% \\
-18.1 \% \\
\end{array}$ & $\begin{array}{l}+10.2 \% \\
-3.2 \% \\
\end{array}$ & $\begin{array}{l}+47.9 \% \\
-40.4 \% \\
\end{array}$ & 10.19 & $\begin{array}{l}+3.8 \% \\
-8.8 \% \\
\end{array}$ & 0.64 & \\
\hline 130 & 5.24 & $\begin{array}{l}+12.8 \% \\
-22.9 \% \\
\end{array}$ & $\begin{array}{l}+21.7 \% \\
-18.6 \% \\
\end{array}$ & $\begin{array}{l}+10.5 \% \\
-3.2 \%\end{array}$ & $\begin{array}{l}+48.0 \% \\
-40.4 \% \\
\end{array}$ & 10.26 & $\begin{array}{l}+3.8 \% \\
-8.7 \% \\
\end{array}$ & 0.54 & $\begin{array}{l}+41.0 \% \\
-39.5 \% \\
\end{array}$ \\
\hline 135 & 4.36 & $\begin{array}{l}+12.4 \% \\
-21.9 \% \\
\end{array}$ & $\begin{array}{l}+21.8 \% \\
-19.0 \% \\
\end{array}$ & $\begin{array}{l}+10.5 \% \\
-3.3 \% \\
\end{array}$ & $\begin{array}{l}+48.5 \% \\
-39.9 \% \\
\end{array}$ & 10.33 & $\begin{array}{l}+3.8 \% \\
-8.8 \% \\
\end{array}$ & 0.45 & \\
\hline 140 & 3.66 & $\begin{array}{l}+11.6 \% \\
-21.5 \%\end{array}$ & $\begin{array}{l}+22.5 \% \\
-19.1 \% \\
\end{array}$ & $\begin{array}{l}+10.2 \% \\
-3.6 \%\end{array}$ & $\begin{array}{l}+46.9 \% \\
-40.2 \% \\
\end{array}$ & 10.39 & $\begin{array}{l}+3.8 \% \\
-8.7 \% \\
\end{array}$ & 0.38 & $\begin{array}{l}+39.8 \% \\
-39.3 \% \\
\end{array}$ \\
\hline 145 & 3.08 & $\begin{array}{l}+11.0 \% \\
-21.2 \% \\
\end{array}$ & $\begin{array}{l}+23.0 \% \\
-19.5 \% \\
\end{array}$ & $\begin{array}{l}+10.4 \% \\
-3.3 \% \\
\end{array}$ & & 10.46 & $\begin{array}{l}+3.7 \% \\
-8.8 \% \\
\end{array}$ & 0.32 & $\begin{array}{l}+39.5 \% \\
-39.4 \% \\
\end{array}$ \\
\hline 150 & 2.60 & $\begin{array}{l}+10.8 \% \\
-20.3 \% \\
\end{array}$ & $\begin{array}{l}+24.1 \% \\
-19.3 \% \\
\end{array}$ & $\begin{array}{l}+10.4 \% \\
-3.4 \%\end{array}$ & $\begin{array}{l}+47.3 \% \\
-39.7 \% \\
\end{array}$ & 10.52 & $\begin{array}{l}+3.7 \% \\
-8.8 \% \\
\end{array}$ & 0.27 & $\begin{array}{l}+39.9 \% \\
-38.9 \% \\
\end{array}$ \\
\hline 155 & 2.20 & $\begin{array}{l}+10.8 \% \\
-20.0 \% \\
\end{array}$ & $\begin{array}{l}+24.3 \% \\
-20.1 \% \\
\end{array}$ & $\begin{array}{l}+10.3 \% \\
-3.5 \%\end{array}$ & $\begin{array}{l}+47.8 \% \\
-39.3 \% \\
\end{array}$ & 10.57 & $\begin{array}{l}+3.8 \% \\
-8.7 \% \\
\end{array}$ & 0.23 & $\begin{array}{l}+40.8 \% \\
-38.2 \% \\
\end{array}$ \\
\hline 160 & 1.88 & $\begin{array}{l}+10.5 \% \\
-20.0 \% \\
\end{array}$ & $\begin{array}{l}+24.9 \% \\
-20.4 \% \\
\end{array}$ & $\begin{array}{l}10.4 \% \\
-3.4 \%\end{array}$ & $\begin{array}{l}+47.8 \% \\
-40.0 \% \\
\end{array}$ & 10.63 & $\begin{array}{l}+3.8 \% \\
-8.7 \% \\
\end{array}$ & 0.20 & $\begin{array}{l}+40.5 \% \\
-39.1 \% \\
\end{array}$ \\
\hline 165 & 1.60 & $\begin{array}{l}+10.6 \% \\
-18.7 \% \\
\end{array}$ & $\begin{array}{l}+25.1 \% \\
-20.6 \%\end{array}$ & $\begin{array}{l}10.4 \% \\
-3.3 \%\end{array}$ & $\begin{array}{l}+49.2 \% \\
-38.5 \% \\
\end{array}$ & 10.68 & $\begin{array}{l}+3.9 \% \\
-8.7 \% \\
\end{array}$ & 0.17 & $\begin{array}{l}+41.8 \% \\
-37.4 \% \\
\end{array}$ \\
\hline 170 & 1.37 & $\begin{array}{l}+10.6 \% \\
-18.4 \% \\
\end{array}$ & $\begin{array}{l}+25.3 \% \\
-21.1 \% \\
\end{array}$ & $\begin{array}{l}+10.5 \% \\
-3.1 \%\end{array}$ & $\begin{array}{l}+49.8 \% \\
-38.7 \% \\
\end{array}$ & 10.74 & $\begin{array}{l}+3.8 \% \\
-8.8 \% \\
\end{array}$ & 0.15 & $\begin{array}{l}+42.4 \% \\
-37.8 \% \\
\end{array}$ \\
\hline 175 & 1.17 & $\begin{array}{l}+10.5 \% \\
-18.2 \% \\
\end{array}$ & $\begin{array}{l}26.0 \% \\
-21.5 \% \\
\end{array}$ & $\begin{array}{l}10.4 \% \\
-3.4 \% \\
\end{array}$ & $\begin{array}{l}+50.5 \% \\
-38.8 \% \\
\end{array}$ & 10.79 & $\begin{array}{l}+3.8 \% \\
-8.7 \% \\
\end{array}$ & 0.13 & $\begin{array}{l}+43.0 \% \\
-37.6 \% \\
\end{array}$ \\
\hline 180 & 1.01 & $\begin{array}{l}+10.3 \% \\
-18.1 \% \\
\end{array}$ & $\begin{array}{l}+26.7 \% \\
-21.6 \%\end{array}$ & $\begin{array}{l}10.4 \% \\
-3.4 \%\end{array}$ & $\begin{array}{l}+50.7 \% \\
-38.6 \% \\
\end{array}$ & 10.84 & $\begin{array}{l}+3.9 \% \\
-8.7 \% \\
\end{array}$ & 0.11 & $\begin{array}{l}+43.2 \% \\
-37.4 \% \\
\end{array}$ \\
\hline 185 & 0.87 & $\begin{array}{l}+10.4 \% \\
-17.6 \% \\
\end{array}$ & $\begin{array}{l}+27.8 \% \\
-21.5 \% \\
\end{array}$ & $\begin{array}{l}+10.3 \% \\
-3.2 \% \\
\end{array}$ & $\begin{array}{l}+51.4 \% \\
-38.6 \% \\
\end{array}$ & 10.90 & $\begin{array}{l}+3.7 \% \\
-8.8 \% \\
\end{array}$ & 0.09 & $\begin{array}{l}+43.8 \% \\
-37.6 \% \\
\end{array}$ \\
\hline 190 & 0.75 & $\begin{array}{l}+10.3 \% \\
-17.3 \% \\
\end{array}$ & $\begin{array}{l}+27.2 \% \\
-22.7 \% \\
\end{array}$ & $\begin{array}{l}+10.4 \% \\
-3.3 \% \\
\end{array}$ & $\begin{array}{l}+52.1 \% \\
-39.2 \% \\
\end{array}$ & 10.95 & $\begin{array}{l}+3.8 \% \\
-8.7 \% \\
\end{array}$ & 0.08 & $\begin{array}{l}+44.4 \% \\
-38.2 \% \\
\end{array}$ \\
\hline 195 & 0.65 & $\begin{array}{l}+10.5 \% \\
-16.2 \% \\
\end{array}$ & $\begin{array}{l}+28.5 \% \\
-22.6 \% \\
\end{array}$ & $\begin{array}{l}+10.1 \% \\
-3.4 \% \\
\end{array}$ & $\begin{array}{l}+52.3 \% \\
-38.9 \% \\
\end{array}$ & 11.00 & $\begin{array}{l}+3.8 \% \\
-8.8 \% \\
\end{array}$ & 0.07 & $\begin{array}{l}+44.7 \% \\
-37.7 \% \\
\end{array}$ \\
\hline 200 & 0.56 & $\begin{array}{l}+10.4 \% \\
-16.3 \% \\
\end{array}$ & $\begin{array}{l}+28.5 \% \\
-23.6 \% \\
\end{array}$ & $\begin{array}{l}+10.6 \% \\
-3.2 \% \\
\end{array}$ & $\begin{array}{l}+53.2 \% \\
-38.9 \% \\
\end{array}$ & 11.04 & $\begin{array}{l}+3.9 \% \\
-8.7 \% \\
\end{array}$ & 0.06 & $\begin{array}{l}+45.8 \% \\
-37.9 \% \\
\end{array}$ \\
\hline
\end{tabular}

Table 2: The production cross sections in the $b \bar{b} \rightarrow A$ process at the Tevatron (in fb) for given $A$ masses (in $\mathrm{GeV}$ ) at a scale $\mu_{F}=\mu_{R}=\frac{1}{4} M_{A}$ with MSTW PDFs. Shown also are the corresponding uncertainties from the various sources discussed as well as the total uncertainty. In the other columns, displayed are the branching ratio $\operatorname{BR}\left(A \rightarrow \tau^{+} \tau^{-}\right)$[in \%] and the product $\sigma(b \bar{b} \rightarrow A) \times \operatorname{BR}\left(A \rightarrow \tau^{+} \tau^{-}\right)$together with their respective total uncertainties. 